\title{
Análise fotoelástica das tensões geradas por transferência em implantes osseointegrados
}

\section{Photoelastic analysis of stress generated by impression techniques in osseointegrated implants}

Caroline Hanada ODO

Gabriela Cassaro de CASTRO

Mestranda em Clínica Odontológica - Área de concentração Prótese Dental - Faculdade de

Odontologia de Piracicaba - UNICAMP - Piracicaba - São Paulo - Brasil.

\section{Rafael Leonardo Xediek CONSANI}

Professor Adjunto da Área de Prótese Total - Departamento de Prótese e Periodontia - Faculdade de Odontologia de Piracicaba - UNICAMP - Piracicaba - São Paulo - Brasil.

\section{Mauro Antonio de Arruda NÓBILO}

Professor Titular da Área de Prótese Fixa - Departamento de Prótese e Periodontia - Faculdade de Odontologia de Piracicaba - UNICAMP - Piracicaba - São Paulo - Brasil .

\begin{abstract}
Resumo
A proposta deste trabalho foi avaliar cinco técnicas de transferência em implantes osseointegrados. Cinco grupos foram testados: GI: transferentes quadrados unidos com resina acrílica Duralay (Reliance. Dental Mfg Co-Worth, IL - EUA) em monobloco; GII: transferentes quadrados unidos com resina acrílica Duralay e seccionamento; GIII: transferentes quadrados unidos com resina acrílica Pattern Resin (GC America - Alsip, IL - EUA) em monobloco; GIV: transferentes quadrados unidos com resina acrílica Pattern Resin e seccionamento; GV: transferentes quadrados unidos com Pattern Resin e haste metálica. Foram utilizados blocos de resina fotoelástica, compostos de dois implantes cada, simulando uma prótese fixa posterior de três elementos. Os transferentes foram entrelaçados com fio dental e, em seguida, unidos com as resinas acrílicas, ou apenas resina e haste metálica. A análise das tensões geradas sobre os implantes foi realizada com o auxílio de um polariscópio circular acoplado a uma câmera digital fotográfica. Foram avaliadas as regiões cervicais e apicais dos implantes por meio de análise qualitativa das franjas fotoelásticas geradas após a união dos transferentes. O grupo que apresentou o maior número de tensões foi o grupo de resina Duralay em monobloco, seguido pelo grupo de resina Pattern Resin em monobloco. Para os demais grupos não foram observadas tensões visíveis a olho nu. Concluiu-se que as técnicas de transferência com reforço metálico e de seccionamento geraram menores tensões sobre os implantes.
\end{abstract}

\section{UNITERMOS}

Prótese parcial fixa; implante dentário endo-ósseo; técnica de moldagem odontológica.

\section{INTRODUÇÃO}

A capacidade de substituir dentes perdidos com implantes osseointegrados tem melhorado a qualidade de vida de muitos pacientes desdentados. Para esses, os implantes proporcionam retenção e estabilidade de próteses parciais ou totais removíveis, reabilitando de forma bastante conservadora pequenos espaços protéticos.

Como a situação biomecânica para um implante é fundamentalmente diferente daquela de um dente natural, que é circundado por ligamento periodontal, a possibilidade de se transferir carga excessiva ao implante e deste ao osso adjacente pode acabar ultra- passando o limite fisiológico e provocar a perda da osseointegração [1]. Portanto, é necessário otimizar a distribuição de carga mastigatória por meio das próteses e implantes para o osso suporte.

Para isso, a existência de excelente adaptação entre o implante e o abutment e deste com a infraestrutura metálica é necessária na determinação do sucesso de uma prótese implanto-suportada [2,3]. Por isso, a adaptação não passiva de infraestrutura ao implante/abutment tem sido sugerida como razão para complicações biológicas e/ou falha dos componentes protéticos.

A falha da adaptação da prótese sobre os abutments é causada por diversos fatores durante o processo de 
confecção de uma prótese implanto-suportada, os quais acabam promovendo a distorção da peça. Dentre estes fatores, podemos citar os procedimentos de moldagem, a confecção do modelo mestre, o padrão de cera da infraestrutura, o processo de fabricação da infraestrutura e o material estético aplicado.

Infraestruturas de próteses convencionalmente realizadas pela técnica da cera perdida para fundição de peça única e que cruzam o arco são imprecisas quanto ao assentamento passivo.

A consequência da falta de uma boa adaptação é a micromovimentação dos componentes protéticos, podendo gerar o rompimento da interface cimentoimplante, ou, nas parafusadas, a perda dos parafusos.

Diante disto, foram revisadas algumas técnicas preconizadas com o intuito de obter-se o melhor assentamento passivo das próteses [3]. Os autores citaram: 1método de verificação da fidelidade do modelo mestre, feito por meio de Index de resina acrílica confeccionado sobre o modelo mestre e prova na boca para verificar adaptação [4]; 2- método de soldagem, no qual as peças são segmentadas, indexadas e soldadas, para se minimizar as distorções de fundição [5]; mais recentemente, 3- método de cimentação dos cilindros da infraestrutura protética pela captura direta na boca [6] e o processo de eletroerosão ou descarga elétrica nas faces internas da infraestrutura, para se obter melhora na interface infraestrutura/abutment [7].

No sistema Branemark existem transferentes cônicos e quadrados que se prestam para transferências e se adaptam aos intermediários e suas réplicas. Porém, existem variações de técnicas para a utilização de tais transferentes, resultando em pesquisas que procuram identificar a superioridade de uma técnica sobre a outra.

Diversos autores preconizaram a união dos transferentes quadrados com resina acrílica para a realização das moldagens de transferência $[8,9]$. No entanto, outras pesquisas demonstraram que a união dos transferentes é desnecessária [10-14].

Em 2002, foi concluído que a precisão promovida por jigs de verificação (Index) não foi superior a procedimentos de moldagem comuns (transferentes cônicos ou quadrados), não melhorando a precisão dos modelos de gesso [15].

Concluiu-se, em 2007, que a técnica do Index obteve maior precisão, assim como a moldagem com os transferentes quadrados com prolongamentos em forma de hélice [16].

Desta forma, a proposta deste trabalho foi identificar técnicas de transferência utilizadas em implantodontia que proporcionem melhores resultados quanto à precisão dimensional.
Assim, reconhecendo a necessidade de maiores esclarecimentos científicos a respeito das técnicas de moldagem, este estudo teve por objetivo analisar as tensões geradas por cinco técnicas de transferência por meio do método da fotoelasticidade.

\section{Material E Método}

Para a realização deste trabalho foi confeccionada uma matriz em aço inoxidável polido nas dimensões $40,0 \mathrm{~mm} \times 15,0 \mathrm{~mm} \times 20,0 \mathrm{~mm}$, onde foram incorporadas réplicas de implantes osseointegrados de diâmetros regulares e configurações hexagonais (Figura 1).

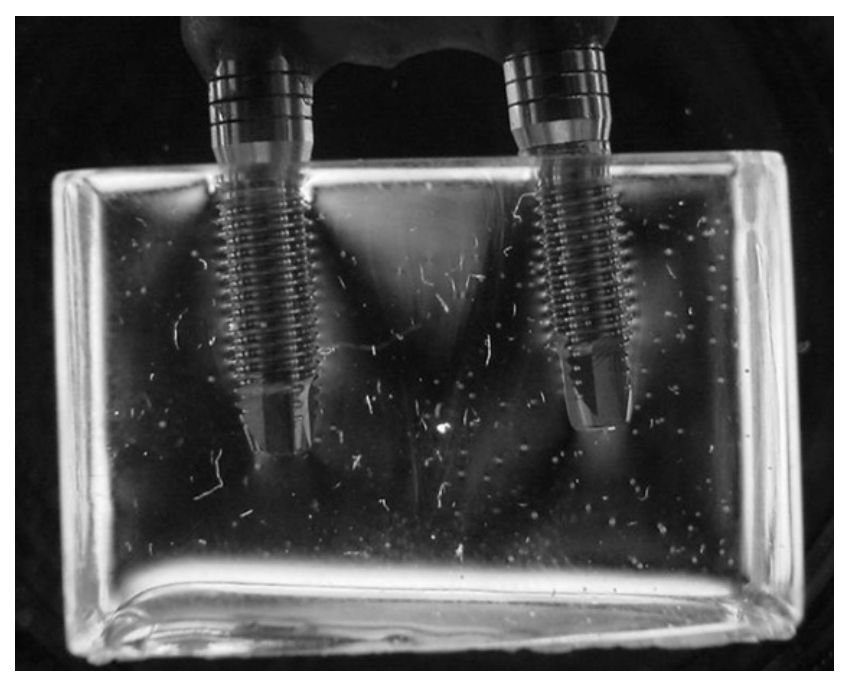

Figura 1 - Vista frontal da matriz metálica.

Sobre as réplicas de implantes foram acoplados transferentes quadrados para implantes de plataforma regular e hexágono externo (Conexão Sistema de Próteses - Arujá, SP, Brasil). Em seguida, o conjunto foi colocado no interior de caixa plástica para a contenção do material duplicador (Figura 2).

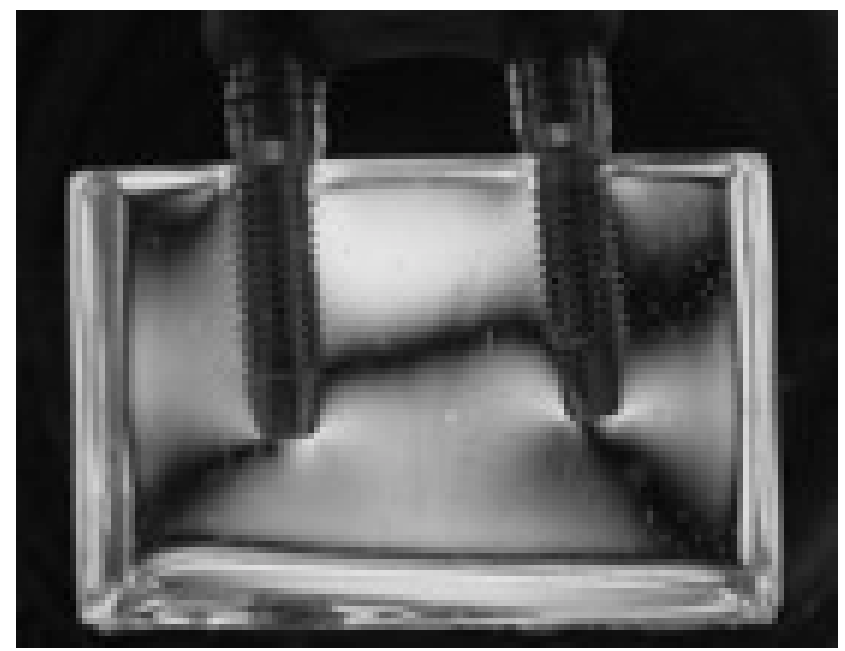

Figura 2 - Transfer acoplado à matriz. 
A duplicação foi realizada com silicone para duplicação (Silicone Master - Talladium Inc.- Valencia, Ca EUA). Concluído o prazo de 24 horas, foram liberados os parafusos dos transferentes e removido o conjunto matriz/transferentes (Figura 3).

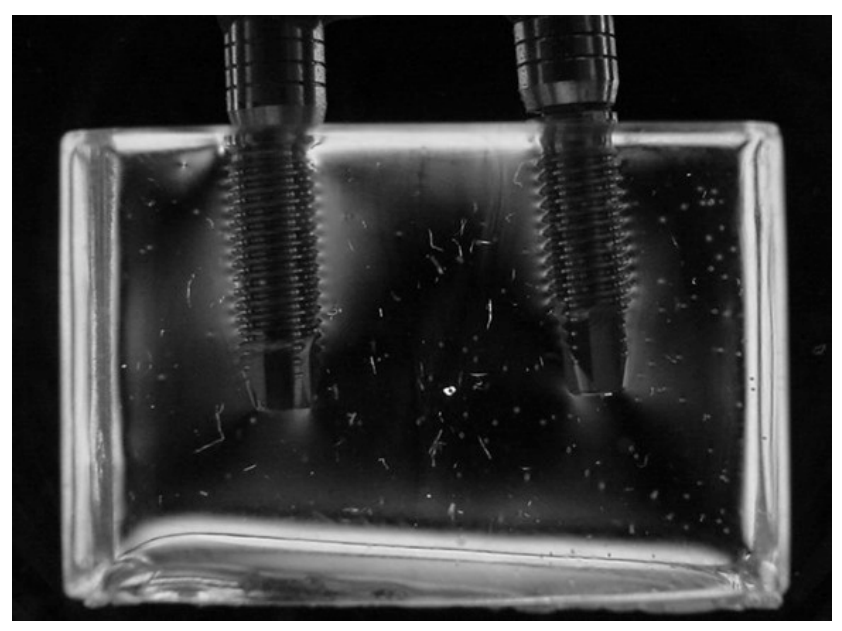

Figura 3 - Molde de silicone com os implantes posicionados.

A partir do molde de silicone foi confeccionado modelo definitivo em resina fotoelástica. Assim, implantes cilíndricos rosqueáveis de 4,0 x 13,0 mm (Master Screw - Conexão Sistema de Próteses - Arujá, SP - BRA) foram cuidadosamente posicionados nos encaixes dos transferentes (Figura 4).

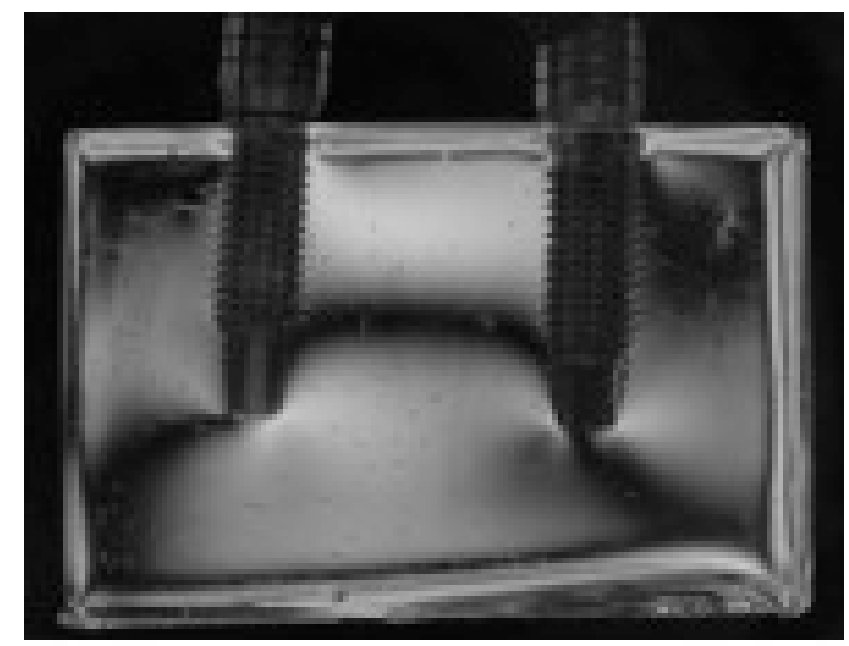

Figura 4 - Modelo de resina fotoelástica com os implantes em posição.

A resina fotoelástica empregada neste estudo foi a resina Araldite (Huntsman - The Woodlands,TX - EUA), que possui dois líquidos: Gy-279 BR, modificado, com fluído reativo, de baixa até média viscosidade, formulado à base de bisfenol $\mathrm{A}$ e um endurecedor HY 2964 à base de amina cicloalifática, modificado, de baixa viscosidade, o que permite melhor aplicação e manuseio. A cura ocorre à tem- peratura ambiente, o que possibilita a confecção de modelos de plástico fotoelástico transparente, de alto brilho e sem exsudação.

A quantidade do material necessária deve ser previamente calculada de acordo com o tamanho da peça a ser modelada, e a proporção da mistura recomendada é de 100 partes de GY279 para 48 de HY2964.

A manipulação da resina foi realizada em Becker, com o auxílio de proveta para a dosagem. Em seguida, a mistura foi levada à câmara acrílica hermeticamente fechada, acoplada a um manômetro e a uma bomba para vácuo; assim, as bolhas de ar incorporadas ao material foram removidas.

Após o vazamento, o molde foi armazenado em recipiente fechado para proteger o material contra impurezas em suspensão durante o período de polimerização de 72 horas, conforme recomendação do fabricante. Para a realização das técnicas de transferência foram utilizados transferentes quadrados para implantes de plataforma regular e hexágono externo (Conexão Sistema de Próteses - Arujá, SP, Brasil).

Este experimento foi dividido em cinco grupos, a saber:

GI: transferentes quadrados unidos com resina acrílica Duralay Reliance. Dental Mfg Co-Worth, IL EUA em monobloco;

- GII: transferentes quadrados unidos com resina acrílica Duralay e seccionamento;

- GIII: transferentes quadrados unidos com resina acrílica Pattern Resin (GC America - Alsip, IL - EUA) em monobloco;

- GIV: transferentes quadrados unidos com resina acrílica Pattern Resin e seccionamento;

- GV: transferentes quadrados unidos com Pattern Resin e haste metálica.

Assim, era-se esperado avaliar a importância da ferulização, do controle da contração de polimerização e da ferulização mais rígida dos componentes protéticos de moldagem.

Os transferentes foram parafusados com chave digital de hexágono externo de $1,17 \mathrm{~mm}$ até sentir resistência e então receberam torque de $10 \mathrm{Ncm}$ com torquímetro protético manual para melhor padronização. Para o grupo de união com resina acrílica apenas, foi realizada uma secção e nova união para minimizar os efeitos da possível contração de polimerização deste material, sendo $\mathrm{N}=1$ para cada grupo analisado.

Mensuração das tensões - Análise fotoelástica

A análise fotoelástica utilizada para este experimento foi a quasitridimensional, realizada com o auxílio de Polariscópio Circular de campo escuro acoplado a uma máquina filmadora digital FD-717 
(Sony - Orange, CA - EUA) que permite visualizar o desenvolvimento das franjas e registrar as imagens em fotografias digitais. As tensões fotoelásticas foram analisadas e comparadas, sendo possível identificar a magnitude das tensões, bem como registrar a proximidade das franjas uma das outras, para avaliar a concentração de tensões.

Para melhor nitidez das imagens, o modelo fotoelástico foi imerso, juntamente com o sistema de transferência, em tanque contendo óleo mineral puro, o que possibilitou ainda melhor visualização das franjas isocromáticas (coloridas).

\section{Forma de análise dos resultados}

Realizou-se registro fotográfico antes do início dos procedimentos referentes a cada grupo experimental, para verificar a presença ou não de tensões no interior do modelo fotoelástico. Em seguida, após o parafusamento dos transferentes de moldagem, novas fotografias foram executadas. Assim, todos os momentos do experimento foram registrados, para posterior avaliação do comportamento das tensões geradas ao redor dos implantes.

A técnica utilizada neste estudo foi uma das formas mais comuns de se determinar a ordem de franja, que é o método qualitativo no qual se utiliza registros fotográficos das amostras para verificação da ordem de franja $(\mathrm{N})$.

- Quanto maior o N, maior a magnitude de tensões;

- Quanto mais próximas as franjas uma das outras, maior a concentração de tensões.

Vale ressaltar que todas as imagens foram analisadas por um mesmo observador e que este desconhecia a proposta do estudo, para que os dados não fossem influenciados nos resultados.

No caso da luz branca, o espectro observado no analisador apresenta colorações típicas para as ordens de franja:

- Franja de ordem $\mathrm{N}=0$ (preta);

- Franja de ordem $\mathrm{N}=1$ (transição Vermelho/ Azul);

- Franja de ordem $\mathrm{N}=2$ (transição Vermelho/ Verde).

A partir deste ponto, todas as franjas de ordens inteiras $(\mathrm{N})$ serão determinadas pela transição: Vermelho/Verde.

Em seguida, as imagens coletadas foram avaliadas e tratadas com o auxílio do software Adobe Photoshop $7.0{ }^{\circledR}$ (Adobe Systems Incorporated - San Jose,CA EUA), com o intuito de facilitar a visualização, compreensão e interpretação, tanto da localização como da intensidade/concentração das tensões distribuídas ao redor dos implantes.

As fotografias foram utilizadas com a finalidade de melhorar a visualização dos resultados obtidos.

\section{Resultados}

As Figuras 5 a 10 representam a análise fotoelástica dos Grupos I a V. Podemos observar o modelo livre de tensão ao início do experimento, anterior às transferências na figura 5 . As técnicas de transferência testadas apresentaram diferentes comportamentos quanto à presença de tensões. Temos então, como observado nas Figuras 7, 9 e 10, que os grupos com seccionamento e haste metálica não apresentaram tensões visíveis a olho nu, enquanto os grupos sem seccionamento, como visto nas figuras 6 e 8 , apresentaram tensões na região apical e na região entre implantes

\section{Análise Fotoelástica: Concentração de Tensões}

\section{Grupo I (Resina Duralay em Monobloco)}

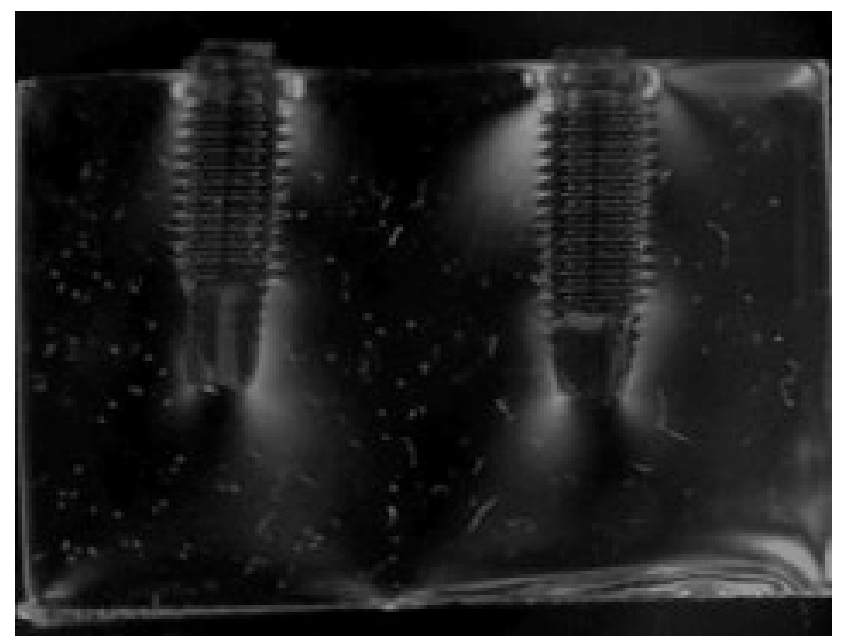

Figura 5. Modelo Fotoelástico livre de tensão.

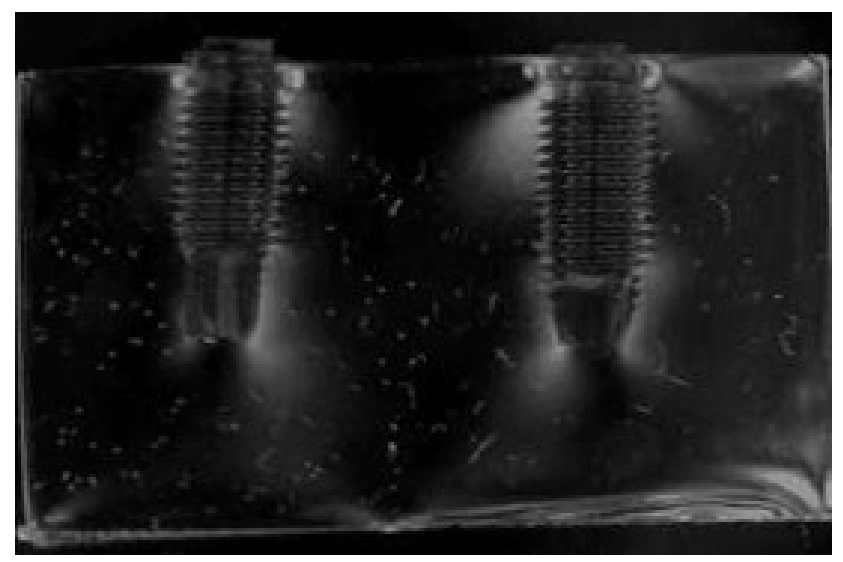

Figura 6 - Modelo fotoelástico com transferência em Duralay em monobloco - Presença de tensão. 


\section{Grupo II (Resina Duralay Seccionada)}

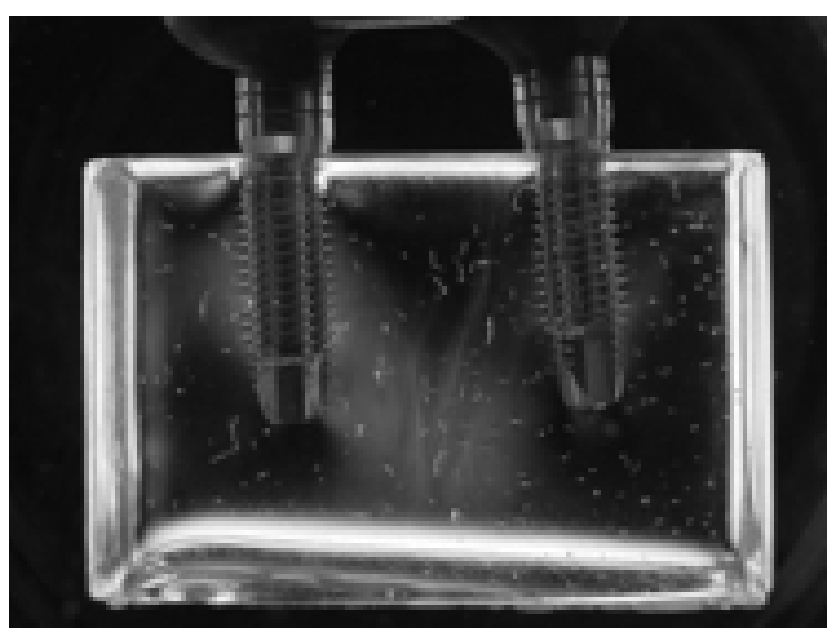

Figura 7 - Modelo Fotoelástico com transferência em Duralay seccionada - Ausência de tensão.

\section{Grupo III (Resina Pattern em Monobloco)}

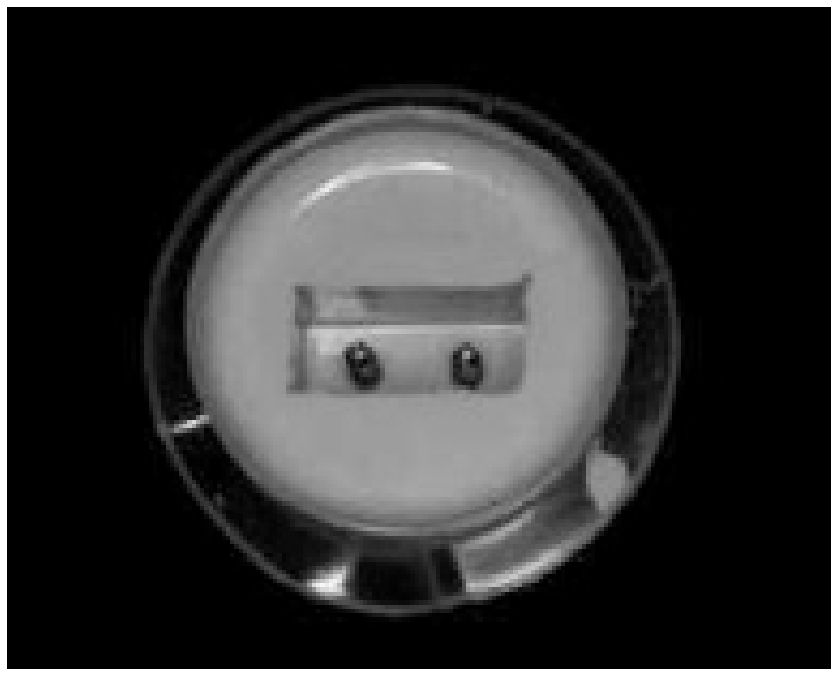

Figura 8 - Modelo Fotoelástico com transferência em Resina Pattern em monobloco - Presença de tensão.

\section{Grupo IV (Resina Pattern Seccionada)}

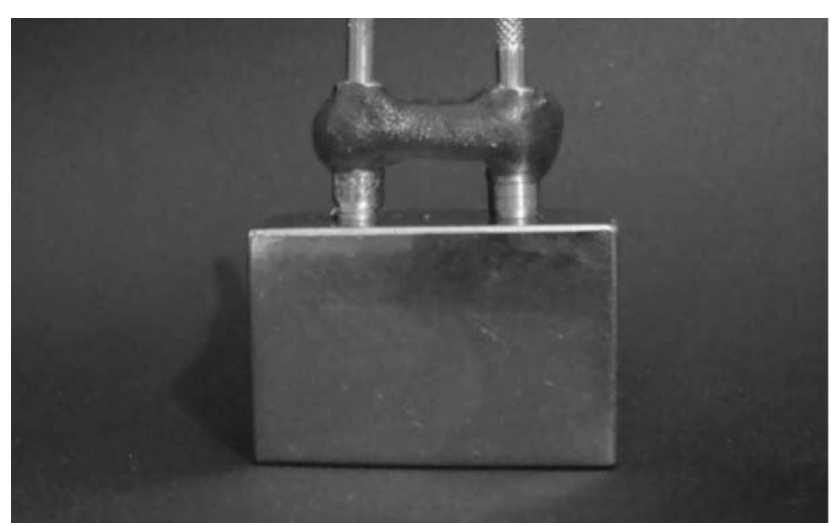

Figura 9 - Modelo Fotoelástico com transferência em Resina Pattern Seccionada - Ausência de tensão.

\section{Grupo V (Resina Pattern e Haste Metálica)}

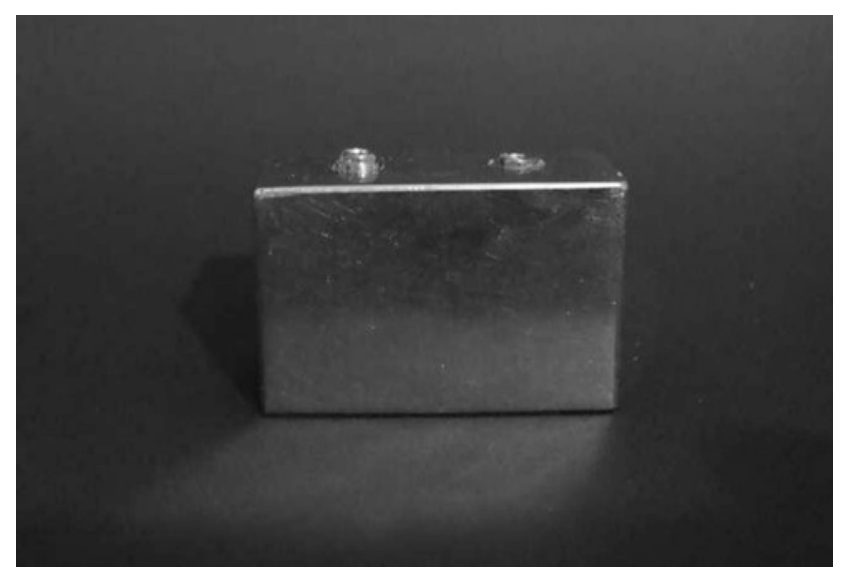

Figura 10. Modelo Fotoelástico com transferência em Resina Pattern e Haste Metálica - Ausência de tensão.

\section{Dıscussão}

A passividade no assentamento de infraestruturas protéticas e o processo de osseointegração são muito enfatizados na literatura [17,18]. Qualquer desalinhamento entre a infraestrutura e os implantes gera tensões na peça protética, nos implantes e no osso adjacente [19], podendo provocar afrouxamento ou quebra dos parafusos protéticos, fratura da prótese ou do implante e, até mesmo, prejudicar a osseointegração $[20,21]$.

Segundo alguns autores, para eliminar as discrepâncias no assentamento, é essencial que o trabalho seja feito em um modelo mestre que reproduza, o mais precisamente possível, a posição dos abutments na boca do paciente [19]. Assim, um importante fator que influencia a precisão de assentamento é a técnica de transferência dos implantes. Neste estudo foi possível observar as tensões fotoelásticas provocadas pelos métodos de transferências nos implantes osseointegrados, as quais podem interferir na passividade da peça protética.

Com o método da fotoelasticidade é possível localizar as tensões ocorridas ao redor dos implantes [22]. Esta técnica para verificar as tensões sobre implantes foi previamente utilizada por diversos autores $[23,24]$.

Materiais de impressão e técnicas empregadas na fabricação dos modelos exercem papel fundamental na adaptação e passividade da estrutura metálica fundida [25]. Inúmeras técnicas de impressão foram sugeridas para alcançar um modelo que permita a passividade e adaptação das próteses sobre implantes. Autores enfatizaram a importância da esplintagem dos transferentes de moldagem na boca, antes do 
registro de impressão [26], sendo essa união apontada como fator primário para obter modelos precisos [8]. Alguns autores questionam a distorção do material de união, o qual pode inserir tensões ao sistema de moldagem $[10,27]$. No presente estudo, foram avaliadas cinco técnicas de transferência utilizando resina acrílica Duralay e Pattern Resin, com e sem seccionamentos, e transferentes unidos com reforço metálico e resina acrílica.

Autores relataram que todas as técnicas de moldagem produziram tensões devido à contração de polimerização dos materiais utilizados para união dos transferentes [13]. Comparando três tipos de materiais para união dos transferentes, autores observaram resultados mais precisos com a utilização de resina acrílica autopolimerizável e gesso para impressão [20]. O seccionamento atua no controle da contração que a resina acrílica sofre ao polimerizar [28]; ao realizar a união com menor volume de material, menor distorção ocorrerá na transferência. De acordo com autores, a resina acrílica Duralay e a resina acrílica Pattern Resin foram eficientes para confecção de Index, contudo a segunda apresentou menor tempo de polimerização [28].

No presente trabalho, podemos observar que os métodos de transferência que exibiram menor tensão na região dos implantes são os de esplintagem com posterior seccionamento e de união com haste metálica (Figuras 7, 9 e 10). Resultados similares também foram apresentados por outros autores [26].

Autores verificaram a precisão de moldagens realizadas com união rígida dos componentes de transferência e resina acrílica com baixa contração de polimerização em mandíbulas edêntulas com cinco implantes e observaram menor distorção com a união rígida dos componentes [29]. Outros autores observaram menor formação de tensão ao unir os trans- ferentes com haste metálica e resina acrílica Pattern Resin [9]. Resultados semelhantes foram encontrados neste trabalho, no qual, a união rígida exibiu menor formação de franjas fotoelásticas.

Os resultados deste estudo sugerem que, quando os transferentes de impressão são conectados com resina acrílica para posterior seccionamento, ou quando se usa haste metálica para a esplintagem dos componentes, podemos obter modelos de trabalho mais precisos, uma vez que menores tensões foram observadas próximas aos implantes. No entanto, a conexão com resina acrílica demanda tempo clínico e sua precisão depende do controle da quantidade de material, espessura, temperatura e do esforço para a retirada do molde da boca. Em termos clínicos, essas variáveis podem ser controladas, mas dependem muito da experiência do operador. Assim, entendemos que, pelo resultado apresentado, a técnica da união com haste metálica mostrou-se de fácil execução, baixo nível de tensão e excelente rigidez, conferindo estabilidade, precisão e confiabilidade à técnica de impressão.

Este estudo objetivou analisar as tensões provocadas por cinco técnicas de transferência utilizadas em implantodontia; todavia, pouco se sabe sobre os valores de tensões aceitáveis clinicamente, sendo necessários mais estudos para a determinação de tais valores.

\section{Conclusão}

Qualitativamente, o grupo que apresentou o maior número de tensões foi o grupo de Resina Duralay em monobloco, seguido pelo grupo de Pattern Resin em monobloco. Para os demais grupos, não foram observadas tensões visíveis a olho nu. Concluiu-se que as técnicas de transferência com reforço metálico e de seccionamento geraram menores tensões sobre os implantes.

\section{ABSTRACT}

The purpose of this study was to evaluate five transfer techniques: GI, square transfer together with en bloc acrylic resin Duralay (Reliance Dental Mfg. Co., Worth, IL, USA); GII, square transfer with acrylic resin Duralay (Reliance Dental Mfg. Co., Worth, IL, USA) and sectioning; GIII, square transfer with en bloc acrylic pattern resin; GIV, square transfer with pattern resin and sectioning; and GV, square transfer with pattern resin and a metal rod. Blocks of photoelastic resin were used, each composed of two implants simulating a posterior fixed prosthesis with three elements. The transferors were intertwined with dental floss and then united with either the acrylic resin or resin combined with a metal rod. The analyses of the stresses generated on the implants were performed with the aid of a circular polariscope coupled with a digital camera. The evaluation was performed on the cervical and apical implants by quality analyses of the photoelastic fringes generated after the union of the transferors. The group with the largest number of stresses was the acrylic resin Duralay en bloc group, followed by the en bloc acrylic pattern resin group. The other groups showed no tensions visible to the naked eye. It was concluded that the transfer techniques with strengthened sectioning and a metal rod generated less tension on the implants.

\section{UNITERMS}

Denture partial fixed; dental implantation endosseous; dental impression technique. 


\section{REFERÊNCIAS}

1. Kenney R, Richards, MW. Photoelastic stress patterns by implant retained overdentures. J Prosthet Dent. 1998; 80:55964.

2. Clelland NL, Gilat A, McGlumphy EA, Brantley WA. A photoelastic and strain gauge analysis of angle abutments of an implant system. Int J oral Maxillofac Implants. 1993;8(5):541-8.

3. Wee AG. Comparison of impression materials for direct multiimplant impression. J Prosthet Dent. 2000; 83:323-31.

4. McCartney JW, Pearson R. Segmental framework matrix: máster cast verification, corrected dast guide, and analog transfer templante for implant-supported prostheses. J Prosthet Dent.1994;71:197-200.

5. Sjogren G, Anderson M, Bergman M. Laser welding of titanium in dentistry. Acta Odont Scand.1988;46:247-56.

6. Jimenes-Lopez V. Reabilitação bucal em próteses sobre implantes. São Paulo: São Paulo; 2000.

7. Contreras EF, Henriques GEP, Giolo SR, Nobilo MAA. Fit of cast commercially pure titanium and Ti-6Al-4V alloy crowns before and after marginal refinement by electrical discharge machining. J Prosthet Dent. 2002 Nov; 88(5):467-72.

8. Assif D, Marshark B, Schmidt A. Accuracy of implant impression techniques. Int $\mathrm{J}$ Oral Maxillofac Implants. 1996;11(2):216-22.

9. Naconecy MM, Teixeira ER, Shinkai RS, Frasca LC, Cervieri A. Evaluation of the accuracy of 3 transfer techniques for implantsupported prostheses with multiple abutments. Int J Oral Maxillofac Implants. 2004; 19(2):192-8.

10. Burawi G, Houston F, Byrne D, Claffey N. A comparison of the dimensional accuracy of the splinted and unsplinted impression techniques for the Bone-Lock implant system. J Prosthet Dent.1997; 77:68-75.

11. Goiato MC, Domitti SS, Consani S. Influência dos materiais de moldagem e técnicas de transferência em implante, na precisão dimensional dos modelos de gesso. J Bras Odontol Clin. 1998;2:45-50.

12. Humphries RM, Yaman P, Bloem TJ. The accuracy of implant master casts constructed from transfer impressions. Int J Oral Maxillofac Implants 1990;5:331-6.

13. Inturregui JA, Aquilino SA, Ryther JS, Lund PS. Evaluation of three impression techniques for osseointegrated oral implants. J Prosthet Dent. 1993; 69:503-9.

14. Phillips KM, Nicholls JI, Ma T, Rubenstein J. The accuracy of three implant impression techniques: A three-dimensional analysis. Int J Oral Maxillofac Implants 1994;9:533-40.

15. De La Cruz JE, Funkenbusch PD, Ercoli C, Moss ME, Graser GN, Tallents RH. Verification jig for implant-supported prostheses: a comparison of standard impression with verification jigs made of differents material. J Prosthet Dent. 2002;88:329-36.

16. Muñoz Chávez A. Avaliação da precisão das técnicas de moldagem para próteses implantossuportadas sobre implantes alinhados e implantes não-alinhados [tese]. Araraquara: Faculdade de Odontologia de Araraquara, UNESP - Univ estadual paulista; 2007.

17. Adell R, Lekholm U, Rockler B, Branemark PI. A 15-year study of osseointegrated implants in the treatment of the edentulous jaw. Int J Oral Surg. 1981; 10:387-416.

18. Branemark PI, Breine U, Adell R, Hansson BO, Lindstrom $\mathrm{J}$, Ohlsson A. Intraosseous anchorage of dental prosthesis, I- experimental studies. Scand J Plast Reconstr Surg. 1969;
3:81-100.

19. Vigolo P, Majzouc Z, Cordioli G. Evaluation of three techniques used for multiple implant abutment impressions. $\mathrm{J}$ Prosthet Dent. 2003; 83(2):186-192.

20. Assif D, Nissan J, Varsano I, Singer A. Accuracy of implant impression splinted techniques: effect of splinting material. Int J Oral Maxillofac Implants. 1999; 14:885-8.

21. Lee H, So JS, Hochstedler JL, Ercoli C. The accuracy of implant impressions: a systematic review. J Prosthet Dent. 2008;100:295-1.

22. Sadoswsky SJ, Caputo A. Effect of anchorage systems and extension base contact on load transfer with mandibular implant-retained overdentures. J Prosthet Dent. 2000; 84:32734.

23. Cehreli M, Duyck J, De Cooman M, Puers R, Naert I. Implant desing and interface force transfer.A photoelastic and stain-gauge analysis. Clin Oral Implant. 2004; 2: 249-57.

24. Machado, ACM. Análise fotoelástica da distribuição de tensão em diferentes sistemas de overdentures sobre implantes osseointegrados [tese]. Piracicaba: Faculdade de Odontologia de Piracicaba, UNICAMP; 2009.

25. Henry PJ. An alternative method for the production of accurate casts and occlusal records in osseointegrated implant rehabilitation. J Prosthet Dent 1987; 58:694-7.

26. Branemark PI, Zab GA, Albrektsson T. Tissue-integrated prostheses. Osseointegration in clinical dentistry. Chicago : Quintessence; 1985. p.11-2, 253-7.

27. Spector MR, Donavan TE, Nicholls JI. An evaluation of impression techniques for osseointegrated implants. J Prosthet Dent. 1900; 63(4):444-7.

28. Cho CG; Chee WWL. Efficient soldering index materials for fixed partial dentures and implant substructure. J Prosthet Dent. 1995;73:424-7.

29. Carr AB, Master J. The accuracy of implant verification casts compared with casts produced from a rigid transfer coping techniques. J Prosthodont. 1996;5:248-52.

Recebido em: 16/08/2010 Aprovado em: 08/02/2011

Correspondência:

Caroline Hanada Odo

Rua Prof Gustavo Pires de Andrade,443 Parque Vila Prudente - SP CEP: 03140-010 carol_odo@fop.unicamp.br 